\title{
Theoretical aspects of High-Speed Supercavitation Vehicle Control
}

\author{
Bálint Vanek, József Bokor and Gary Balas
}

\begin{abstract}
A control system based on feedback linearization is developed for a high-speed supercavitating underwater vehicle. The supercavitation bubble surrounding the body leads to reduced drag but is also responsible for the undesired switched, nonlinear and delay dependent behavior caused by the phenomena known as planing. The theoretical contributions of the switched control design are discussed in connection with the mathematical description of the system. Special attention is made to understand and handle the complex and novel dynamics of the vehicle.
\end{abstract}

\section{PROBLEM DESCRIPTION}

Based on the recent advancements [8], [6], [7] in simulation and control of a High-Speed Supercavitating Underwater Vehicle (HSSV), a new mathematical model was developed [1] to capture more details of the vehicle dynamics. The nonlinear interaction of the body with the cavity wall, showing memory effect, is very important, hence the way the cavity surface is described (cavity shape is a function of the history of the vehicle motion and cavitator area) plays an important role in the vehicle dynamics (Fig. 1). The theoretical aspects, i.e. controller design, controllability and tracking, raised by the novel system type are discussed in the following sections. The layout of the paper is as follows: a brief description of the generalized vehicle model developed in [1] is presented in Sec.II followed by the basic overview of the proposed control methodology (Sec.III). Section IV describes the theoretical design and controllability properties, including a solution for the reference signal tracking. Simulation results are presented in Section V. The future direction of this research and conclusions are presented in Sec.VI.

\section{MODEL DESCRIPTION}

The system equations for the longitudinal motion of the HSSV are written in a local tangent reference frame attached to the nose of the vehicle (Fig. 2). The states in the statespace equations are: $z(t)[\mathrm{m}]$ nose vertical position; $\theta(t)[\mathrm{rad}]$ body pitch angle; $w(t)[\mathrm{m} / \mathrm{s}]$ vertical speed; and $q(t)[\mathrm{rad} / \mathrm{s}]$ body pitch rate. The two control inputs are $\delta_{\text {cav }}$ and $\delta_{\text {fin }}$ the cavitator and fins deflection. In addition to the gravity force $\left(F_{g}\right)$ another force $\left(F_{p}\right)$ caused by the contact of the vehicle with the fluid surface can be present. It depends on the relative immersion depth $\left(h^{\prime}\right)$ and immersion angle $(\alpha)$ of the transom. Due to the lack of space the details of the system equations for the HSSV are omitted but the reader is referred to [1] for further details. The overall system equations can be written as:

$\dot{x}(t)= \begin{cases}A x(t)+B u(t)+F_{g} & \text { if } c^{T}(\delta) x(t) \leq 0, \\ A x(t)+F_{p}(t, x, \delta)+B u(t)+F_{g} & \text { if } c^{T}(\delta) x(t) \geq 0,\end{cases}$

This work was supported by the ONR, award number N000140110229, Dr. Kam Ng Program Manager

B.Vanek, J.Bokor and G.Balas are with University of Minnesota,

J.Bokor is on sabbatical leave from Hungarian Academy of Sciences

Corresponding author: B. Vanek $\{$ vanek $\mathrm{e} a \mathrm{em}$. umn .edu $\}$

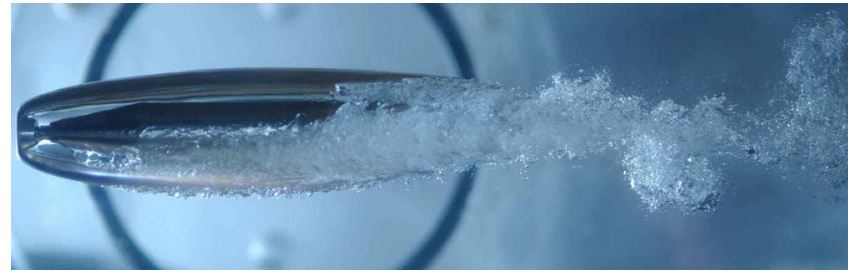

Fig. 1. Water tunnel experiment on supercavitation

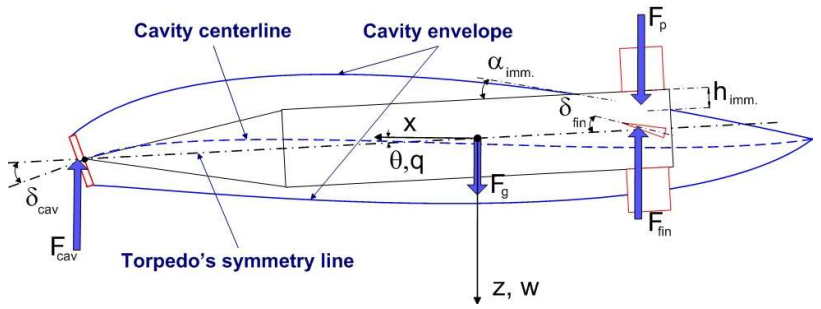

Fig. 2. The underwater vehicle with all the variables in the longitudinal plane during immersion

where $x(t) \in \mathcal{X} \subset \mathbb{R}^{4}, x_{0}=x\left(t_{0}\right), u(t) \in \mathbb{R}^{2}, x^{T}(t)=$ $[\hat{z}(t), \theta(t), w(t), q(t)], \hat{z}(t)=z(t)+R-R_{c}$, and $c^{T}(\delta)=$ $[1-\delta, 0, L, 0]$ with $\delta$ denoting the delay operator: $\delta x(t)=$ $x(t-\tau)$. Using this notation, $F_{p}(t, x, \delta)$ can be written as:

$$
\begin{gathered}
F_{p}(x, \delta)=P\left(1-\frac{R^{\prime}}{h^{\prime}(x, \delta)+R^{\prime}}\right)^{2}\left(\frac{1+h^{\prime}(x, \delta)}{1+2 h^{\prime}(x, \delta)}\right) \alpha(x, \delta), \\
h^{\prime}(x, \delta)= \begin{cases}R^{-1} c^{T}(\delta) x(t) & \text { if } c^{T}(\delta) x(t) \leq 0, \\
0 & \text { if } c^{T}(\delta) x(t) \geq 0,\end{cases} \\
\alpha(x, \delta)= \begin{cases}c_{\alpha}^{T}(\delta) x(t)-V^{-1} \dot{R}_{c} & \text { if } c^{T}(\delta) x(t) \leq 0, \\
0 & \text { if } c^{T}(\delta) x(t) \geq 0,\end{cases}
\end{gathered}
$$

where $c_{\alpha}^{T}=[0,1, \delta / v, 0]$.

Eqs.1-4 describe the system as a bimodal, switched system. In the first (linear) mode the vehicle is flying inside the cavity and in the second mode it is planing e.g. on the bottom (or top) of the cavity. Note the characteristics of the switched system: (i) the switching hyperplane depends on the delayed state variable $x(t-\tau)$, (ii) in the first mode the system dynamics is linear and in the second mode it is nonlinear input affine, i.e. the control inputs effect the dynamics linearly in both modes, and (iii) the switching condition does not depend on the control inputs. The reachability (controllability) properties of this system are important for control design.

\section{CONTROLLER DESIGN CONSIDERATIONS}

The longitudinal axis HSSV model has linear and nonlinear time delay dynamics with three distinct modes: free 
flying, planing at the bottom and at the top of the cavity. The planing conditions are linear and define a switching hyper plane that separates the dynamic modes. Controllability results for bimodal switching systems are available for linear case under very specific conditions only [4]. To make use of linear controllability results, the approach taken in this paper is to apply different feedback laws in each modes to transform the system to linear time invariant, with the assumption of full state measurement. The control law design is synthesized in a new multivariable canonic coordinate frame. Extending the controllability results on bimodal switched LTI systems to time delayed switching conditions, requires the analysis of time delayed zero dynamics. The tracking problem is solved using a multivariable pole placement [1] extension of dynamic inversion.

Given that the input variables enter linearly in both modes, and all the states are measurable (assumption): it is possible to select two outputs such that the relative degrees are identical in both modes.

This allows the system to be transformed into LTI canonical form using linear state feedback in the central mode (1) and nonlinear state-delayed feedback in the planing mode $(2,3)$. Hence the state equations in both modes can be linearized using a similar structure.

After feedback linearization, the system has identical dynamics in both modes. This implies that the dynamics are continuous on the switching surface $c(\delta) x=0$. To analyze controllability, the zero dynamics of $\left(c(\delta), A_{c}, B_{c}\right)$ have to be computed. The state space is time dependent due to the delay dependent switching condition. Using both inputs, the zero dynamics will be controllable. The tracking problem can be solved using multivariable pole placement extension of the switching dynamic inversion controllers.

\section{THEORETICAL BACKGROUND OF THE CONTROLLER DESIGN}

Our approach relies on the assumption that the delay in the equations of motion can be eliminated by applying suitable feedback. Then the controllability analysis and the control design can be performed for the bimodal LTI system. Since the concept of relative degree plays a central role in this approach, its definition for nonlinear, time delay and LTI systems is given.

\section{A. Feedback linearization}

Consider a nonlinear input affine system:

$$
\begin{aligned}
& \dot{x}=f(x)+\sum_{i=1}^{m} g_{i}(x) u_{i}, \quad x \in \mathcal{X}, \quad u \in \mathcal{U} \\
& y_{j}=h_{j}(x), \quad y_{j} \in \mathcal{Y}, \quad j=1, \ldots, p,
\end{aligned}
$$

Definition 1: The system has a vector relative degree $r=\left[r_{1}, \ldots, r_{p}\right], r_{i} \geq 0, \forall i$, if at a point $x_{0}$

(i) $L_{g_{j}} L_{f}^{k} h_{i}(x)=0, \ldots j=1, \ldots, m, k<r_{i-1}$,

(ii) The matrix

$$
\mathcal{A}_{I A}=\left[\begin{array}{ccc}
L_{g_{1}} L_{f}^{r_{1}-1} h_{1}(x), & \ldots, & L_{g_{1}} L_{f}^{r_{1}-1} h_{1}(x) \\
\vdots & & \\
L_{g_{m}} L_{f}^{r_{p}-1} h_{p}(x), & \ldots, & L_{g_{m}} L_{f}^{r_{p}-1} h_{p}(x)
\end{array}\right]
$$

has rank $p$ at $x_{0}$.

For linear time invariant (LTI) systems given by $(A, B, C)$, we have that $L g_{j} L_{f}^{r_{i}-1} h_{i}(x)=c_{i} A^{r_{i}-1} b_{j}$ and if $p=m$ then the vector relative degree is defined if $\operatorname{rank} \mathcal{A}_{L T I}=$ $m$. The concept of relative degree can be extended to time delay systems, too. Usually this is defined for a discrete time equivalent of the continuous time systems by introducing the discrete time shift operator $\delta$ as $\delta x_{t}=x_{t-\tau}$ with $\tau$ denoting the given time delay. The time delay system is given now by $(A(\delta), B(\delta), C(\delta))$, i.e. the matrices depend on the delay operator. This implies that the coefficients are elements of the polynomial ring $\mathcal{R}[\delta]$. The relative degree is defined similarly to the LTI case as follows.

Definition 2: Given the single input - single output linear time delay system $(A(\delta), b(\delta), c(\delta))$. It has relative degree $r>0$ if $c A^{k} b=0, \quad k=0, \ldots, r-1$ and $c A^{r} b \neq 0$. It has pure relative degree $r$ if in addition $c A^{r} b$ is an invertible element of $\mathcal{R}[\delta]$.

This definition has an obvious extension to the multivariable case. It requires the matrix $A(\delta)$ to be invertible over $\mathcal{R}^{p \times p}[\delta]$. To perform the analysis and design a controller, new state variables for equation 1 are chosen to be as $\bar{x}=T_{c} x$,

$$
\left[\begin{array}{l}
\bar{x}_{1}(t) \\
\bar{x}_{2}(t) \\
\bar{x}_{3}(t) \\
\bar{x}_{4}(t)
\end{array}\right]=\left[\begin{array}{c}
z(t) \\
-V \theta(t)+w(t) \\
\theta(t) \\
q(t)
\end{array}\right]
$$

The matrix used for this coordinate transformation is:

$$
T_{c}=\left[\begin{array}{c}
c_{1}^{\top} \\
c_{1}^{\top} A \\
c_{2}^{\top} \\
c_{2}^{\top} A
\end{array}\right]=\left[\begin{array}{cccc}
1 & 0 & 0 & 0 \\
0 & -V & 1 & 0 \\
0 & 1 & 0 & 0 \\
0 & 0 & 0 & 1
\end{array}\right]
$$

where the first two states were considered as output variables. $\left(C=\left[\begin{array}{cc}c_{1} & c_{2}\end{array}\right]\right)$

The state space equations in the new coordinate system are:

$$
\dot{\bar{x}}= \begin{cases}A_{c} \bar{x}(t)+B_{c} u(t)+\bar{F}_{g} & \text { if } \bar{c}^{T}(\delta) \bar{x}(t) \leq 0, \\ A_{c} \bar{x}(t)+\bar{F}_{p}(t, x, \delta)+B_{c} u(t)+\bar{F}_{g} & \text { if } \bar{c}^{T}(\delta) \bar{x}(t) \geq 0,\end{cases}
$$

where

$$
A_{c}=\left[\begin{array}{cccc}
0 & 1 & 0 & 0 \\
-\alpha_{110} & -\alpha_{111} & -\alpha_{120} & -\alpha_{121} \\
0 & 0 & 0 & 1 \\
-\alpha_{210} & -\alpha_{211} & -\alpha_{220} & -\alpha_{221},
\end{array}\right] B_{c}=\left[\begin{array}{c}
0 \\
c_{1}^{\top} A B \\
0 \\
c_{2}^{\top} A B
\end{array}\right]_{(11)}
$$

The difference between $F_{\text {grav }}$ and $\bar{F}_{\text {grav }}$ is that $F_{\text {grav }}=$ $\left(T_{c} \cdot F_{\text {grav }}\right)+C_{1}$ where $C_{1}$ is a constant associated with the shift in the origin of the coordinate system. Similarly $\bar{F}_{\text {plane }}=\left(T_{c} \cdot F_{\text {plane }}\right)$.

Note that the inputs enter linearly in the state equations in both modes. In addition, it is assumed that all states can be measured. This allows us to select two outputs defined as $y_{1}=x_{1}$ and $y_{2}=x_{3}$ constituting an output matrix $C_{c}$, such that there exists pure vector relative degree in both modes, and in addition, these are identical. The relative degree for the modes are:

$$
\begin{aligned}
& r_{1}^{1}=2, \quad r_{2}^{1}=2, \quad r_{1}^{1}+r_{2}^{1}=n=4 \quad \text { Mode } 1 \\
& r_{1}^{2}=2, \quad r_{2}^{2}=2, \quad r_{1}^{2}+r_{2}^{2}=n=4 \quad \text { Mode } 2
\end{aligned}
$$


The consequence of this property is that one can apply state feedback in both modes such that it eliminates time delay in Mode 1 and nonlinearity (exact feedback linearization) in Mode 2. This feedback is given by:

$u_{f l c}=\left\{\begin{array}{l}M_{1}^{-1}\left(\dot{y}_{13}(t)-F_{\alpha} x(t)-\bar{F}_{g}+v_{I}(t)\right. \\ \text { if } c^{T}(\delta) x(t) \leq 0 \\ M_{1}^{-1}\left(\dot{y}_{13}(t)-F_{\alpha} x(t)-\bar{F}_{g}-\bar{F}_{p}(x, \delta)+v_{I I}(t)\right. \\ \text { if } c^{T}(\delta) x(t) \geq 0,\end{array}\right.$

where $M_{1}=(C A B), y_{13}=\left[y_{1}, y_{3}\right]^{T}$, and the feedback gain $F_{\alpha}$ is defined by the controllability invariants $\alpha_{i j k}, \quad i=1,2, \quad j=1,2, \quad k=0,1$ of the linear part $A, B$ of the system (Eq.11).

The feedback linearized closed loop has the following form in both modes:

$$
\dot{x}_{c}=\left[\begin{array}{llll}
0 & 1 & 0 & 0 \\
0 & 0 & 0 & 0 \\
0 & 0 & 0 & 1 \\
0 & 0 & 0 & 0
\end{array}\right] x_{c}+\left[\begin{array}{ll}
0 & 0 \\
1 & 0 \\
0 & 0 \\
0 & 1
\end{array}\right]\left[\begin{array}{l}
v_{1} \\
v_{2}
\end{array}\right]
$$

and the switching condition is given by the sign of $y_{s}=$ $c(\delta) x_{c}$.

\section{B. Controllability analysis of the bimodal system}

The controllability of the linearized bimodal dynamics has to be analyzed and a tracking controller designed. Results on controllability of single input single output LTI systems with single switching surface and relative degrees $r=r_{1}=r_{2}=$ 1 has been published by Heemels et al. [4].

In their approach the problem is reduced to analyzing the dynamics of the system on the switching surface. This is given by the zero dynamics derived with respect to the "switching output" $y_{s}$. It was shown that the zero dynamics have to be controllable when using positive $y_{s}$ in one mode (negative $y_{s}$ in the second mode, respectively). Heemels et al. assumed that the system is both left and right invertible and the dynamics are continuous on the switching surface, i.e. $A_{1} x+b_{1} u=A_{2} x+b_{2} u$.

The same results can be obtained using the following simple reasoning. Since the relative degree $r=1$, under the above assumptions, the zero dynamics can be written as

$$
\dot{\eta}(t)=H \eta+ \begin{cases}g_{1} y_{s}(t) & \text { if } y_{s}(t) \leq 0 \\ g_{2} y_{s}(t) & \text { if } y_{s}(t) \geq 0\end{cases}
$$

with $\eta \in \mathbb{R}^{n-1}$.

To compute the reachability subspace of the zero dynamics, the Lie-algebra of the vector spaces $H \eta+g_{1} y_{s}^{+}$and $H \eta+g_{2} y_{s}^{-}$need to be defined. $y_{s}^{+}, y_{s}^{-}$denotes a positive (respectively negative) control to $\left(H, g_{1}\right)$ and $\left(H, g_{2}\right)$. It is simple to show that this is the Lie - algebra generated by $H x+\left[g_{1}, g_{2}\right] y_{s}, y_{s}^{T}=\left[y_{s}^{+}, y_{s}^{-}\right]$and this is given (at $\left.\eta=0\right)$ by the vectors $\left\{\left[g_{1}, g_{2}\right], H\left[g_{1}, g_{2}\right], \ldots, H^{n-2}\left[g_{1}, g_{2}\right]\right\}$. Denote this subspace by $\mathcal{R}\left(H,\left[g_{1}, g_{2}\right]\right)$. Thus a necessary condition for controllability is that $\mathcal{R}\left(H,\left[g_{1}, g_{2}\right]\right)=\mathbb{R}^{n-1}$, i.e., the pair $\left(H,\left[g_{1}, g_{2}\right]\right)$ has to be controllable. This is a Kalman - like rank condition, since in a given mode one can use only positive (respectively negative) control in the zero dynamics, imposing an additional condition on $H$. A sufficient condition is that if $H$ has an even number of eigenvalues with zero real parts, then the zero dynamics are controllable with nonnegative inputs. More results on controllability with nonnegative inputs can be found in [3], [9]. This result is extended for our application as follows.

Consider the MISO system with $B \in \mathbb{R}^{n \times m}$ and $y_{s}=C x$. Also consider the case, when there is a direction $p \in \operatorname{Im}\{B\}$ such that the system is left and right invertible corresponding to the direction $p$. Using the notation $B=[p \bar{B}]$, one has the system:

$$
\dot{x}=A x+p u_{p}+\bar{B} \bar{u}, \quad y_{s}=C x .
$$

Let us denote by $\mathcal{V}^{*}$ the largest $(A, p)$ - invariant subspace in $\mathcal{C}=\operatorname{ker}\{C\}$ and by $\mathcal{W}_{*}$ the smallest $(C, A)$ invariant subspace over $\operatorname{Im}\{p\}$. It follows that system has the following decomposition induced by a choice of basis in $\mathcal{V}^{*}$ and $\mathcal{W}_{*}$ :

$$
\begin{aligned}
\dot{\xi} & =A_{11} \xi+\gamma v \\
u_{p} & =\frac{1}{\gamma}\left(-A_{12} \eta-\bar{B}_{21} \bar{u}+v\right) \\
\dot{\eta} & =A_{22} \eta+\bar{B}_{22} \bar{u}+G y_{s},
\end{aligned}
$$

Since $r=1, \xi=y_{s}$, equation (20) describes the dynamics of the system on $\mathcal{C}$. Rewriting the zero dynamics equation as

$$
\dot{\eta}=P \eta+Q \bar{u}+R y_{s}
$$

assuming that $Q$ is monic.

Proposition 1: If the pair $(P, Q)$ is controllable, then $\eta$ is controllable "without" using $y_{s}$, e.g. by applying $\bar{u}=$ $Q^{\#}\left(-R y_{s}+w\right)$. If the pair $(P, Q)$ is not controllable, then the conditions of controllability with unconstrained $\bar{u}$ but nonnegative $y_{s}$ is

1) The pair $(P,[Q R])$ has to be controllable.

2) Consider the decomposition induced by the reachability subspace $\mathcal{R}(P, Q)$,

$$
\begin{array}{cc}
\dot{\eta}_{1}= & P_{11} \eta_{1}+P_{12} \eta_{2}+Q \bar{u}+R_{1} y_{s} \\
\dot{\eta}_{2}= & P_{22} \eta_{2}+R_{2} y_{s},
\end{array}
$$

where $R_{2} \neq 0$. Then the imaginary part of the eigenvalues of $P_{22}$ cannot be zero.

Remark 1: The first condition is a Kalman-rank condition. The second one can be given in some alternative forms using e.g. results from [3], [9].

For the high speed supercavitating vehicle model, this result has to be applied to a time delay system. The following approach is taken.

Since only one delay time is present in the switching condition, it is possible to discretize the system with an extended state space by including the delayed state variable. Since feedback linearization has been already applied, it is possible to use a backward difference scheme defined for LTI systems that preserves the geometry needed to analyze the zero dynamics. The discrete time state equations are:

$$
x(t+1)=A_{d} x(t)+B_{d} v(t), \quad y_{s}=C_{d} x(t)
$$

where

$$
\begin{aligned}
A_{d}=\left[\begin{array}{ccccc}
1 & T & 0 & 0 & 0 \\
0 & 1 & 0 & 0 & 0 \\
0 & 0 & 1 & T & 0 \\
0 & 0 & 0 & 1 & 0 \\
1 & 0 & 0 & 0 & 0
\end{array}\right], \quad B_{d}=\left[\begin{array}{cc}
0 & 0 \\
\beta_{21} T & \beta_{22} T \\
0 & 0 \\
\beta_{41} T & \beta_{42} T \\
0 & 0
\end{array}\right], \\
C_{d}=[1,0, L, 0,-1]
\end{aligned}
$$


with $T$ denoting the sample time.

The next step is to find the relative degrees by selecting one of the inputs, $v_{1}$ for example. They are identically $r=2$ for both modes since the feedback linearization and state transform resulted in the same linear canonic form in both modes.

To obtain the zero dynamics one has to construct a state transform matrix $T_{c d}$ from the row vectors spanning the orthogonal complement of $\mathcal{V}^{*}$ and $\operatorname{Im}\left\{B_{d 1}\right\}$ where $\operatorname{Im}\left\{B_{d 1}\right\}$ is the first column of $B_{d}$.

It can be shown that $\mathcal{V}^{* \perp}=\operatorname{span}\left\{c_{s}, c_{s} A_{d}\right\}$ and the remaining 3 rows of $T_{c d}$ is selected from $i m B_{1}^{\perp}$ resulting in the transform:

$$
T_{c d}=\left[\begin{array}{ccccc}
\multicolumn{5}{c}{c_{s}} \\
\multicolumn{5}{c}{c_{s} A} \\
1 & 0 & 0 & 0 & 0 \\
0 & 0 & 1 & 0 & 0 \\
0 & \beta_{41} T & 0 & -\beta_{21} T & 0
\end{array}\right]
$$

Using this state transform $\left[\xi^{T}(t), \eta^{T}(t)\right]^{T}=T_{c d} x(t)$ and that $\mathcal{V}^{*}$ is $\left(A_{d}, B_{d 1}\right)$ invariant, leads to the following decomposition:

$$
\begin{array}{r}
\xi(t+1)=\left[\begin{array}{ll}
0 & a_{12} \\
0 & a_{22}
\end{array}\right] \xi(t)+\left[\begin{array}{ccc}
0 & 0 & 0 \\
0 & e_{22} & e_{23}
\end{array}\right] \eta(t)+ \\
+\left[\begin{array}{c}
0 \\
b_{21}
\end{array}\right] v_{1}(t)+\left[\begin{array}{c}
0 \\
f_{22}
\end{array}\right] v_{2}(t) \\
y_{s}=\left[\begin{array}{ll}
1 & 0
\end{array}\right] \xi(t) \quad \text { switching condition } \\
\eta(t+1)=P \eta(t)+R \xi(t)+Q v_{2}(t)
\end{array}
$$

where

$$
P=\left[\begin{array}{ccc}
p_{11} & p_{12} & p_{13} \\
0 & p_{22} & p_{23} \\
0 & 0 & p_{33}
\end{array}\right], R=\left[\begin{array}{cc}
0 & r_{12} \\
0 & r_{22} \\
0 & 0
\end{array}\right], Q=\left[\begin{array}{c}
0 \\
0 \\
q_{31}
\end{array}\right]
$$

The zero dynamics are described by the last equation. (The same approach can be repeated when selecting the second column of $B_{d}$.) Using Proposition 1, it can be seen that due to their special structure, the $(P, Q)$ pair is controllable. This implies that the dynamic inversion controller with switching and an pole placement for tracking error stability can be applied to control the bimodal system.

\section{Multivariable Pole Placement for Tracking}

The performance objective of the control design is to track desired state commands. The inversion based controller has the following form:

$$
\begin{gathered}
{\left[\begin{array}{l}
u_{1}(t) \\
u_{2}(t)
\end{array}\right]=(C A B)^{-1}\left(\left[\begin{array}{l}
\dot{x_{1}}(t) \\
\dot{x_{2}}(t)
\end{array}\right]_{r e f}-\left[\alpha_{u}\right]\left[\begin{array}{l}
x_{1}(t) \\
x_{2}(t)
\end{array}\right]-\right.} \\
\left.-\left[\alpha_{l}\right]\left[\begin{array}{l}
x_{3}(t) \\
x_{4}(t)
\end{array}\right]-\left[G_{c}\right]-\left[P_{c}(t, \tau)\right]-\left[\begin{array}{l}
v_{1}(t) \\
v_{2}(t)
\end{array}\right]\right)
\end{gathered}
$$

The reference tracking part of the controller:

$$
\left[\begin{array}{l}
v_{1}(t) \\
v_{2}(t)
\end{array}\right]=\left[\bar{\alpha}_{u}\right]\left[\begin{array}{l}
x_{1}(t)-x_{1, r e f}(t) \\
x_{2}(t)-x_{2, r e f}(t)
\end{array}\right]+\left[\bar{\alpha}_{l}\right]\left[\begin{array}{l}
x_{3}(t)-x_{3, \text { ref }}(t) \\
x_{4}(t)-x_{4, \text { ref }}(t)
\end{array}\right]
$$

$$
\left[\alpha_{u}\right]=\left[\begin{array}{ll}
-\alpha_{110} & -\alpha_{111} \\
-\alpha_{210} & -\alpha_{211}
\end{array}\right]\left[\alpha_{l}\right]=\left[\begin{array}{ll}
-\alpha_{120} & -\alpha_{121} \\
-\alpha_{220} & -\alpha_{221}
\end{array}\right]
$$

$$
\left[\bar{\alpha}_{u}\right]=\left[\begin{array}{cc}
-\bar{\alpha}_{110} & -\bar{\alpha}_{111} \\
0 & 0
\end{array}\right]\left[\bar{\alpha}_{l}\right]=\left[\begin{array}{cc}
0 & 0 \\
-\bar{\alpha}_{220} & -\bar{\alpha}_{221}
\end{array}\right]
$$

The feedback linearized closed-loop has the following form in all modes:

$$
\begin{gathered}
\dot{x}_{c}=\left[\begin{array}{cccc}
0 & 1 & 0 & 0 \\
-\alpha_{110} & -\alpha_{111} & -\alpha_{120} & -\alpha_{121} \\
0 & 0 & 0 & 1 \\
-\alpha_{210} & -\alpha_{211} & -\alpha_{220} & -\alpha_{221}
\end{array}\right] x_{c}- \\
-B_{c} B^{-1}\left(\left[\begin{array}{llll}
-\alpha_{110} & -\alpha_{111} & -\alpha_{120} & -\alpha_{121} \\
-\alpha_{210} & -\alpha_{211} & -\alpha_{220} & -\alpha_{221}
\end{array}\right] x_{c}+\left[\begin{array}{l}
v_{1} \\
v_{2}
\end{array}\right]\right) \\
A_{c l}=A_{c}-B_{c} F_{i n v}+B_{c} F_{c t r}= \\
{\left[\begin{array}{cccc}
0 & 1 & 0 & 0 \\
-\bar{\alpha}_{110} & -\bar{\alpha}_{111} & 0 & 0 \\
0 & 0 & 0 & 1 \\
0 & 0 & -\bar{\alpha}_{220} & -\bar{\alpha}_{221}
\end{array}\right]}
\end{gathered}
$$

The closed-loop system is stable for a given set of $\bar{\alpha}$ coefficients. With feedback linearization, the system behaves the same regardless of the interior switching state, hence one linear outer loop controller can guarantee stability and appropriate tracking properties for the complex system. A variety of linear design approaches can be used for that purpose [5], [2], but the focus of this paper is on the feedback linearization controller. Hence a simple pole-placement controller is synthesized as the outer-loop tracking controller. The inner loop dynamics after feedback linearization, using the new canonical coordinates are:

$$
\left[\begin{array}{l}
\dot{x}_{1} \\
\dot{x}_{2} \\
\dot{x}_{3} \\
\dot{x}_{4}
\end{array}\right]=\left[\begin{array}{llll}
0 & 1 & 0 & 0 \\
0 & 0 & 0 & 0 \\
0 & 0 & 0 & 1 \\
0 & 0 & 0 & 0
\end{array}\right]\left[\begin{array}{l}
x_{1} \\
x_{2} \\
x_{3} \\
x_{4}
\end{array}\right]+B_{c}\left[\begin{array}{c}
\Delta \delta_{f} \\
\Delta \delta_{c}
\end{array}\right]
$$

where $\Delta \delta_{f, c}$ denotes the additional deflection of the fins and cavitator commanded by the pole-placement controller. The objective is to place the closed-loop poles to obtain the desired tracking response. This system is nilpotent, because all eigenvalues of $A$ are zero. The first two states relative to vertical position and speed are controlled by the fins $\left(\Delta \delta_{f}\right)$ and the other two states relative to vehicle angle and angle rate are controlled by the cavitator due to the lack of cross coupling. The feedback linearization controller is denoted by $F_{i n v}$ and the tracking controller by $\Delta \delta_{f}$ and $\Delta \delta_{c}$ respectively. The resulting reference tracking controller has the following form:

$$
\begin{gathered}
{\left[\begin{array}{l}
\Delta \delta_{f} \\
\Delta \delta_{c}
\end{array}\right]=(C A B)^{-1}\left\{\left[\begin{array}{cc}
-\bar{\alpha}_{110} & -\bar{\alpha}_{111} \\
0 & 0
\end{array}\right]\left[\begin{array}{l}
x_{1}(t)-x_{1, r e f}(t) \\
x_{2}(t)-x_{2, r e f}(t)
\end{array}\right]+\right.} \\
\left.+\left[\begin{array}{cc}
0 & 0 \\
-\bar{\alpha}_{220} & -\bar{\alpha}_{221}
\end{array}\right]\left[\begin{array}{l}
x_{3}(t)-x_{3, \text { ref }}(t) \\
x_{4}(t)-x_{4, \text { ref }}(t)
\end{array}\right]+\left[\begin{array}{l}
\dot{x}_{2, r e f} \\
\dot{x}_{4, r e f}
\end{array}\right]\right\}
\end{gathered}
$$

The closed-loop has the following dynamics:

$$
A_{c l}=A_{c}-B_{c} F_{i n v}+B_{c}\left[\begin{array}{l}
\Delta \delta_{f} \\
\Delta \delta_{c}
\end{array}\right]
$$

The tracking part of the controller is responsible for the location of the poles. The eigenvalues of the system are:

$$
\begin{gathered}
\lambda_{1,2}=-0.5 \bar{\alpha}_{221} \pm 0.5 \sqrt{\left(-\bar{\alpha}_{221}\right)^{2}-4 \bar{\alpha}_{220}} \\
\lambda_{3,4}=-0.5 \bar{\alpha}_{121} \pm 0.5 \sqrt{\left(-\bar{\alpha}_{121}\right)^{2}-4 \bar{\alpha}_{120}}
\end{gathered}
$$

which are stable based on the selection of the $\bar{\alpha}$ coefficients. 


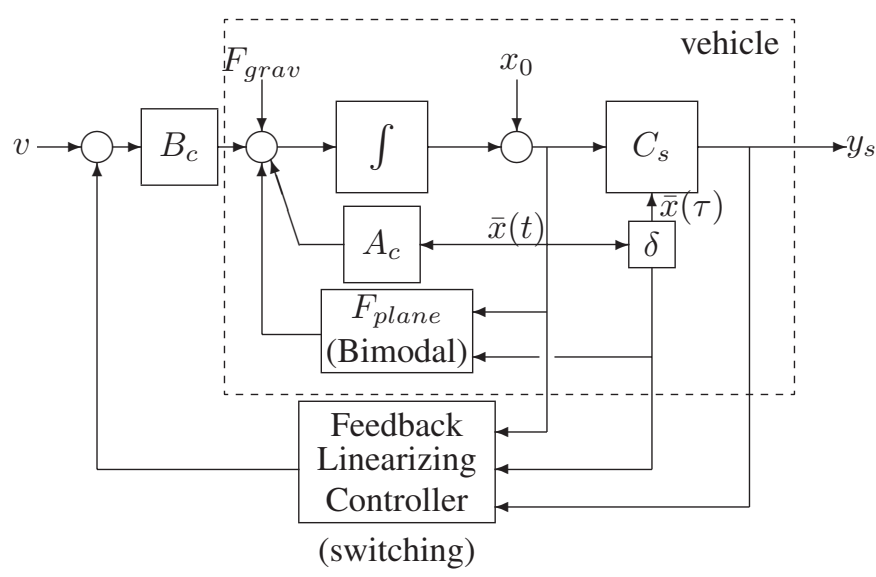

Fig. 3. Inner loop: Switching feedback linearizing controller

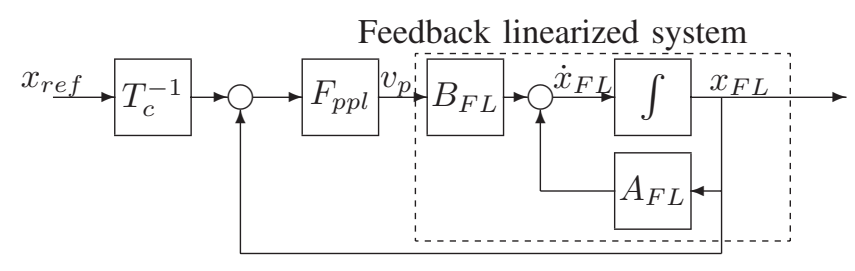

Fig. 4. Outer loop: Inversion controller for tracking

The structure of the feedback controller can be seen in Figure 3-4. The inner-loop feedback linearizes the system and the outer-loop provides reference tracking. Different controllers are used in the 3 switched modes. They are selected by a switching logic based on the planing model and measurements. The cavity wall is noisy and it is the switching surface. Hence the outer-loop must be robust to handle that "noise." After feedback linearization the reference tracking part needs only to be designed for a linear model. It is possible to track both position, velocity, angle and angle rate commands with different weights if they are selected consistently. The designed pole placement controller also operates on the transformed canonic coordinates. The special structure of the feedback linearized system enables control of position and angle without cross coupling.

\section{Control of a Supercavitating Vehicle Model}

Simulations are performed in MATLAB/SIMULINK environment and parameter dependencies are analyzed in comparison with a reference setup. The maneuver is an obstacle avoidance maneuver: the horizontal speed is constant $75 \mathrm{~m} / \mathrm{s}$ while the vehicle moves up $17.5 \mathrm{~m}$ and returns to continue its straight path as seen in Figure 6.

It is assumed that the water conditions (pressure, temperature, viscosity etc.) are constant during the 4 second maneuver. A noise component is added to the cavity wall resulting in an uncertain switching surface. The cavity wall varies between $90-110 \%$ of the nominal cavity gap. The noise is modeled as a random white noise process passing through a filter $G_{n}=\frac{1}{600 s+1}$. Another simplification in the original problem is the lack of actuator model. It is included in the simulation setup with dynamics: $G_{a c t}=\frac{1}{200 s+1}$. The performance specifications are to track trajectory reference commands and reduce limit cycle oscillations. The reference
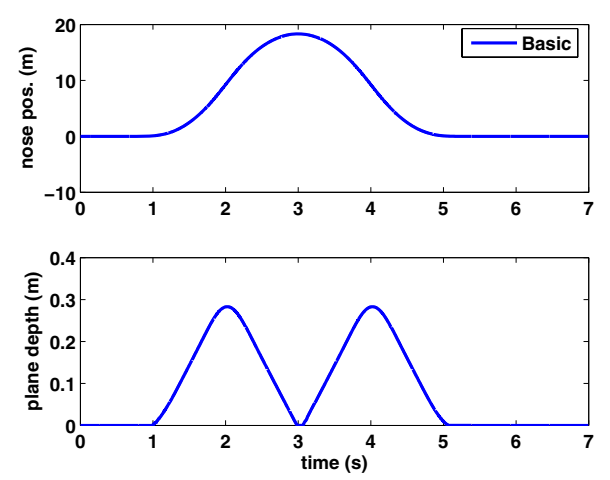

Fig. 5. Simulated trajectory of high speed supercavitating vehicle
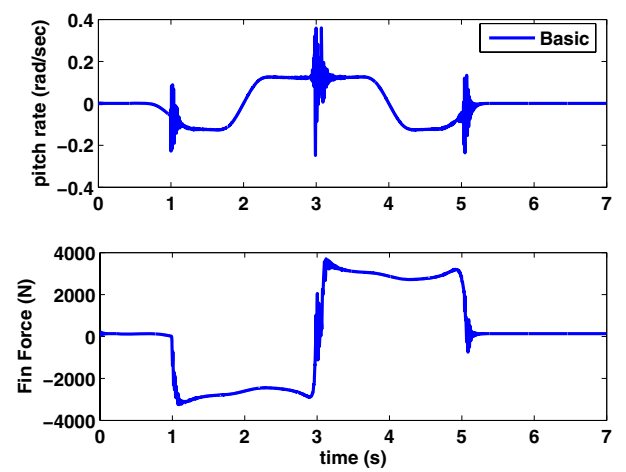

Fig. 6. Control deflection and pitch rate with basic setup

tracking properties received the higher priority as compared with the damping the oscillatory behavior. The following controller gains were selected: $-\bar{\alpha}_{110}=-40000 ;-\bar{\alpha}_{111}=$ $-400 ;-\bar{\alpha}_{220}=-90000 ;-\bar{\alpha}_{221}=-600$ With which the resulting eigenvalues are $-300 ;-300 ;-200 ;-200$.

The resulting contribution from the tracking part of the controller with these high gains is still negligible comparing with the inversion based contribution to compensate against the effect of planing.

The basic actuator model is: $G_{a c t}=\frac{1}{200 s+1}$ which has bandwidth $30 \mathrm{~Hz}$, noticeably slower actuators are not able to stabilize the system, while faster actuators has better performance, with less oscillation. The knowledge about delay time also plays an important role in the controller performance. The vehicle tracks the reference signal when the uncertainty is small, but oscillations grow due to imprecise knowledge of the delay, immersion into the liquid is getting deeper and lasts longer, which results in instability when the uncertainty in delay time exceeds approximately 20 percent.

The noise model can be modified two different ways: magnitude and filter. The maximum planing depth remains the same but the actuator deflection is more radical if the noise has larger magnitude, it has larger spikes and has longer settling times, Figure 8.

Changing the noise filter leads to different characteristic if it is faster than the sampling time of the sensors than it has no effect, if it is slow enough than the controller can hardly deal with it, it leads to larger planing depths and oscillations. The control signals change less rapidly and have smaller spikes. The simulation (Fig.9) shows a large magnitude noise 

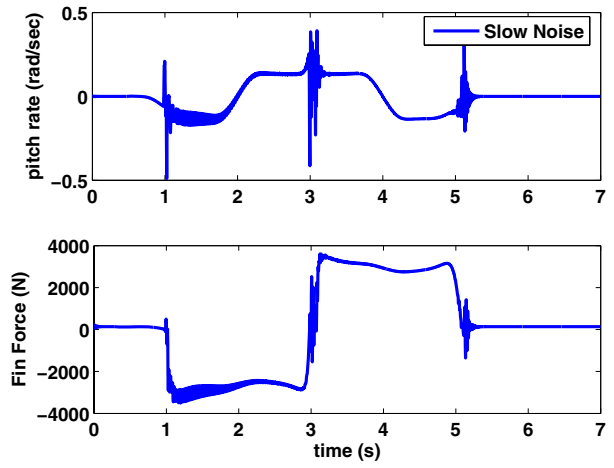

Fig. 7. Trajectory tracking: Slow cavity noise
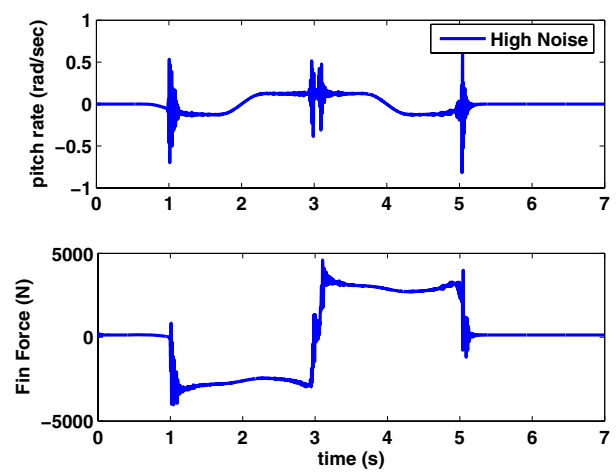

Fig. 8. Trajectory tracking: High cavity noise

with $R_{c} \pm 0.5(R c-R)$ cavity radius and a slow noise case when the noise filter is $G_{n}=\frac{1}{60 s+1}$.

As expected the maximum planing depth increases as the maneuver become more radical. It is interesting to note that the control signals do not follow the same trend. The fin deflection remain basically the same, while the cavitator deflection has small contribution from the increased planing, possibly because the planing angle is different in the different cases.

\section{Summary AND Future Research}

Supercavitation is a very promising way to increase the speed of underwater vehicles at the expense of a complicated vehicle architecture. Successful development of such a system will require increased collaboration between fluid and control researchers. As an intermediate step the control design challenges including delayed state dependency, nonlinearities and switching with noisy switching surface were analyzed and an inversion based control methodology was proposed on a recently developed 2-DOF mathematical model of the HSSV.

The ultimate goal for the future research is implementation of a three dimensional trajectory tracking controller on the HSSV test vehicle. The 3-D motion will no longer have a symmetry plane, hence the asymmetric fin immersion and non-vertical planing forces leading to non input affine systems require special attention. Furthermore robust constraint fulfillment remains an open issue, which can be attempted to solve by RHC control based methods.
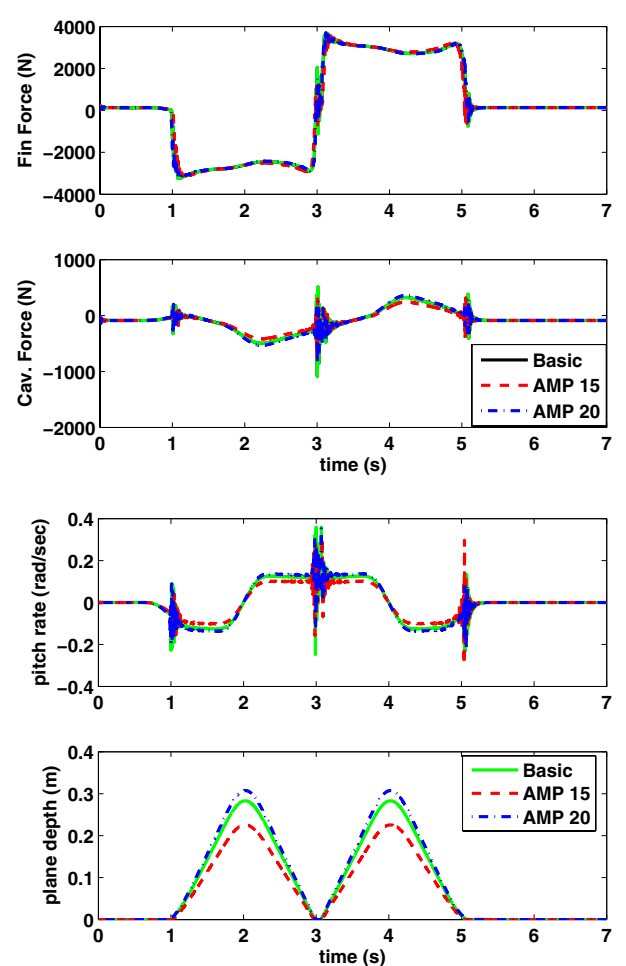

Fig. 9. Trajectory tracking: $15,18,20 \mathrm{~m}$ amplitude maneuvers

\section{REFERENCES}

[1] Gary J. Balas, József Bokor, Bálint Vanek, and Roger E.A. Arndt. Control of Uncertain Systems: Modelling, Approximation, and Design, chapter Control of High-Speed Underwater Vehicles, pages 25-44. LNCIS. Springer-Verlag, 2006.

[2] G.J. Balas, R. Chiang, A.K. Packard, and M. Safanov. Robust control toolbox. MUSYN Inc. and The MathWorks, Natick MA, 2005.

[3] R.F. Brammer. Controllability in linear autonomous systems with positive controllers. SIAM J. Control, 10:329-353, 1972.

[4] M.K. Camlibel, W.P.M.H. Heemels, and J.M. Schumacher. On the controllability of bimodal piecewise linear systems. In:Alur R, Pappas GJ (eds.) Hybrid Systems: Computationand Control LNCS 2993, Springer, Berlin, 250-264, 2004.

[5] J.C. Doyle, K. Glover, P. Khargonekar, and B. Francis. State-space solutions to standard $\mathcal{H}_{2}$ and $\mathcal{H}_{\infty}$ control problems. IEEE Trans Auto Control 34:831-847, 1989.

[6] J. Dzielski and A. Kurdila. A benchmark control problem for supercavitating vehicles and an initial investigation of solutions. Journal of Vibration and Control, 9(7):791-804, 2003.

[7] I.N. Kirschner, D.C. Kring, A.W. Stokes, and J.S. Uhlman. Contro strategies for supercavitating vehicles. J Vibration and Control 8:219242, 2002.

[8] I.N. Kirschner, B. J. Rosenthal, and J.S. Uhlman. Simplified dynamical systems analysis of supercavitating high-speed bodies. In Fifth International Symposium on Cavitation (CAV2003), Osaka,Japan, 2003.

[9] S.H. Saperstone and J.A. Yorke. Controllability of linear oscillatory systems using positive controls. SIAM J. Control, 9:253-262, 1971. 